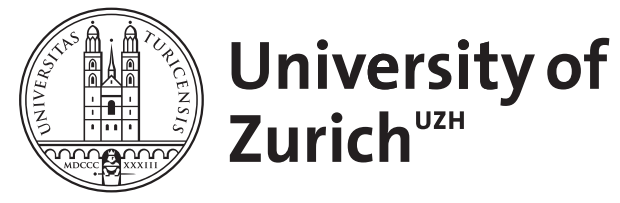

Zurich Open Repository and Archive

University of Zurich

University Library

Strickhofstrasse 39

CH-8057 Zurich

www.zora.uzh.ch

Year: 2018

Wie können wir die Tabak-Exposition am besten messen?

Sudano, Isabella

DOI: https://doi.org/10.1024/1661-8157/a003024

Posted at the Zurich Open Repository and Archive, University of Zurich

ZORA URL: https://doi.org/10.5167/uzh-165749

Journal Article

Published Version

Originally published at:

Sudano, Isabella (2018). Wie können wir die Tabak-Exposition am besten messen? Praxis, 107(14):745.

DOI: https://doi.org/10.1024/1661-8157/a003024 


\section{Wie können wir Tabak-Exposition (b) am besten messen?}

Der Übersichtsartikel von Gruber und Schuurmans [1] fasst Literatur zu Biomarkern, die zur Bewertung der aktiven und passiven Exposition gegenüber Tabak/Nikotin verwendet werden, inklusive allgemein empfohlener Cut-off-Werte mit klinischer Relevanz.

Die objektive Quantifizierung des Expositionsstatus Aktiv-/Passiv-Raucher ist entscheidend für die Dokumentation des Tabakgebrauchs, die Schätzung des Bevölkerungsrisikos und der durch Rauchen bedingten Krankheitslast sowie für die Bewertung der Fortschritte von Tabak-Kontrollprogrammen.

Darüber hinaus spielen Biomarker zur Überwachung der Tabak-/Nikotinbelastung und deren gesundheitlichen Auswirkungen eine entscheidende Rolle bei der Regulierung von Tabakprodukten und in der Gesundheitspolitik.

Zur Bewertung der Raucherprävalenz und Passivrauchbelastung werden am häufigsten standardisierte Fragebögen verwendet. Der selbst angegebene aktive Tabakkonsum und die passive Exposition gegenüber Tabak oder Nikotin kann jedoch häufig unpräzise sein.

Aus diesen Gründen wurden biologische Marker von Tabakkonsum in Prävalenzstudien und epidemiologischen Studien verwendet, um Raucherstatus zu validieren und die Exposition der Bevölkerung gegenüber Tabak zu schätzen und zu überwachen [2].

Als Biomarker zählen die Konzentrationen von rauchbezogenen Komponenten, deren Metabolite sowie die Produkte, die durch die Interaktion zwischen Tabak-Chemikalien und Zielmolekülen in biologischen Materialien entstehen [3].

Die Konzentrationen dieser Biomarker werden durch Aufnahme-, Verteilungs-, Metabolisierungs- und Eliminierungsprozesse, sowie von Krankheiten (z.B. Asthma, COPD, Niereninsuffizienz) oder physiologische Konditionen (z.B. Schwangerschaft) beeinflusst.

In einer Population, die Raucher und Nichtraucher umfasst, wäre die ideale Verteilung der für Tabak spezifischen Biomarker bimodal über Nichtraucher und Raucher.

So einfach ist es in der Realität nicht: Die meisten Biomarker haben keine hohe Sensitivität und/oder Spezifität. Dies führt zu einer gewissen Anzahl von falsch-negativen Ergebnissen, und, streitbar noch relevanter, von falschpositiven, hauptsächlich aufgrund von Umwelt-Faktoren.

Sensitivität und Spezifität können durch eine korrekte Auswahl von Cut-off-Werten optimiert werden: Im Allgemeinen erreicht ein niedrigerer Cut-off-Wert eine höhere Sensitivität auf Kosten einer geringeren Spezifität (eine höhere Anzahl von Nichtrauchern weist Biomarker-Level über dem Cut-off auf). Umgekehrt ist ein höherer Cut-off mit hoher Spezifität und niedrigerer Sensibilität verbunden.
Cotinin (in Urin, Plasma oder Speichel gemessen) wird häufig als Standard für die Klassifizierung des Expositionsstatus verwendet $[4,5]$. Als Bestimmung der Langzeitexposition auf Tabak/Nikotin wird auch die Konzentration von Cotinin im Haar gebraucht [6].

Andere gut untersuchte Biomarker zur Messung des Tabakkonsums und der Tabakentwöhnung sind das ausgeatmete Kohlenmonoxid, Kohlenmonoxid-Hb und Thiocyanat [7].

Gruber und Schuurmans [1] haben die aktuell verfügbaren Daten zum Stellenwert von Cotinin im Urin, Plasma und Speichel zusammengefasst; sie kommen dabei zur Schlussfolgerung, dass idealerweise landesspezifische Cut-off-Werte und Cut-off-Werte für unterschiedliche Kategorien (d. h. aktive Raucher vs. passive Raucher, passive Raucher im Vergleich zu Nichtrauchern) wünschenswert seien.

\section{Bibliografie}

1. Gruber D, Schuurmans M: Biomarker für Rauchen - Welche Cut-off-Werte sind anwendbar? Praxis 2018; 107: 758-762.

2. Benowitz NL: Biomarkers of environmental tobacco smoke exposure. Environ. Health Perspect 1999; 107: 349-355.

3. Kim S: Overview of cotinine cutoff values for smoking status classification. Int J Environ Res Public Health 2016; 13: 1236.

4. National Research Council: Biologic markers of pulmonay toxicology. Washington DC; National Academy Press: 1989.

5. Murray RP, Connett JE, Lauger GG, Voelker HT: Error in smoking measures: Effects of intervention on relations of cotinine and carbon monoxide to self-reported smoking. The Lung Health Study Research Group. Am J Public Health 1993; 83: 12511257.

6. Etter JF, Vu DT, Perneger TV: Saliva cotinine levels in smokers and nonsmokers. Am J Epidemiol 2000; 151: 251-258.

7. Florescu A, Ferrence R, Einarson T, Selby P, Soldin O, Koren G: Methods for quantification of exposure to cigarette smoking and environmental tobacco smoke: focus on developmental toxicology. Ther Drug Monit 2009; 31:14-30.

8. SRNT Subcommittee on Biochemical Verification. Biochemical verification of tobacco use and cessation. Nicotine Tob Res 2002; 4: 149-159.

PD Dr. med Isabella Sudano

Hypertonie-, Lipid-, Tabakentwöhnungsprechstunde

Kardiovaskuläre Forschung

Kardiologie

Universitäres Herzzentrum

Universitätsspital

Rämistrasse 100

8091 Zurich

isabella.sudano@usz.ch 